\title{
S2.5
}

\section{TBE: UNA PROBLEMATICA EMERGENTE. EPIDEMIOLOGIA, CLINICA E DIAGNOSI DI LABORATORIO}

\section{Martelli P., Crovatto M.}

SOS Immunologia Cinica e Virologia

AO Santa Maria degli Angeli

Via Montereale 33170 Pordenone

TBE è una delle più pericolose infezioni umane del sistema nervoso, presente in gran parte del Nord, Centro, Est Europa e del Nord dell'Asia.

Manca una reale conoscenza della diffusione dell'infezione anche se appare evidente la comparsa di nuovi focolai in zone precedentemente indenni.

Si stima che in Russia colpisca circa 11000 soggetti ogni anno e nelle altre zone d' Europa almeno 3000. In Italia sono stati segnalati casi in Toscana, in Trentino, in Veneto , nel Sud Tirolo e in FVG.

L'agente eziologico è il TBE virus, gruppo Tick Borne Flavivirus (sottogruppo: Mammalian), famiglia Flaviviridae, genere Flavivirus L'infezione viene trasmessa dal morso di zecca infetta (Europa Ixodes ricinus; Asia Ixodes persulcatus ). Meno frequente e associato in piccoli cluster epidemici è il contagio tramite latte non pastorizzato. I picchi di presenza dei vettori nell'ambiente (Maggio-Giugno; Settembre-Ottobre) sono seguiti dai picchi di incidenza annuale della malattia. I primi sintomi compaiono infatti mediamente dopo 7-14 giorni dopo il morso che solo il 50 - 68\% dei Pazienti ricorda.

L'infezione può decorrere asintomatica o dare quadri clinici di diversa gravità dalla forma febbrile alla grave mengoencefalomielite.

Decisivo per la diagnosi è l'apporto delle indagini microbiologiche. La ricerca virale sia mediante coltura

che RT PCR da sangue e/o liquor può fornire informazioni preziose solo in fase precoce e assume rilevanza in particolare nella diagnosi differenziale con le altre infezioni trasmesse da morso di zecca all'esordio. Quando compaiono i sintomi neurologici il virus non è più evidenziabile nel sangue e nel liquor, mentre nella maggioranza dei casi sono presenti gli anticorpi.

Il metodo di scelta per la ricerca rapida di IgG e IgM in siero e liquor è enzyme linked immunosorbent assay (ELISA). Nella valutazione dei risultati è tuttavia necessaria cautela 